\title{
OUTPUT FEEDBACK COMPENSATORS FOR WEAKENED ANTI-WINDUP OF ADDITIVELY PERTURBED SYSTEMS
}

\author{
Sergio Galeani ${ }^{*, 1}$ Salvatore Nicosia* \\ Andrew R. Teel ${ }^{* *, 2}$ Luca Zaccarian ${ }^{*, 1}$ \\ * Dip. di Informatica, Sistemi e Produzione \\ Univ. di Roma "Tor Vergata", 00133 Roma (Italy) \\ [galeani, zack] @disp. uniroma2. it \\ ** Electronic and Computer Engineering Dept. \\ Univ. of California, Santa Barbara, 93106 CA (USA) \\ teel@ece.ucsb.edu
}

\begin{abstract}
It was recently shown that certain instances of the natural anti-windup problem necessarily lead to control systems lacking robust "in the large" stability, and that in such instances it is meaningful to solve a weakened anti-windup problem (trading performance for robust stability). While previously proposed solutions of the weakened problem essentially require state feedback, this paper provides output feedback solutions for the case of additively perturbed systems. Copyright (C)2005 IFAC
\end{abstract}

Keywords: Anti-windup, control system design, control nonlinearities, saturation, feedback control, stability robustness, nominal performance.

\section{INTRODUCTION}

The presence of input saturation nonlinearities in otherwise linear closed loop systems can cause dramatic performance losses known as "windup" effects. Anti-windup compensation techniques denote modifications to the closed-loop aimed at reducing as much as possible such losses. The first anti-windup techniques date back to the 1950's, even before the advent of digital control systems. Initial results on application specific solutions were soon followed by results of much more general applicability with formal stability guarantees (see (Hanus, 1988; Kothare et al., 1994) for sur-

\footnotetext{
1 Partially supported by MIUR (COFIN: "Robustness and optimization techniques for high performance control systems", FIRB: "Tiger") and ASI/ARS (I/R/277/02, $\mathrm{I} / \mathrm{R} / 125 / 02)$.

2 Partially supported by AFOSR grant number F4962003-1-0203 and grant number ARO DAAD19-03-1-0144, NSF under Grant ECS-0324679.
}

veys of these early schemes). In the last decade, several advanced techniques have been proposed for the design of anti-windup compensators providing formal stability and performance guarantees and often arising from optimality based synthesis algorithms (see, e.g., (Grimm et al., 2003; Kothare et al., 1994; Mulder et al., 2001; Peng et al., 1998) and references therein).

As pointed out in (Teel and Kapoor, 1997a), the rigorous definition of a natural anti-windup problem entails two requirements: a) the closed loop trajectories must not be modified as long as saturation is inactive; b) input/output stability between certain signals must be achieved.

In the presence of uncertainty on the controlled plant, a "natural" robust anti-windup problem can be defined by simply requiring (a) and (b) to hold for all uncertainties in a suitable family (usually, a set of perturbations having incremental gain smaller than a given value); such an approach 
has been considered e.g. in (Turner et al., 2004; Grimm et al., 2004; Saeki and Wada, 2002), where both analysis and synthesis results are given.

However, it was shown in (Galeani and Teel, 2004) that requirement (a) can impair the robustin-the-large (i.e., for all uncertainties in an a priori given family) achievement of requirement (b), even when a robust-in-the-large, globally stabilizing controller is available for the saturated perturbed plant; this motivated the definition of a relaxed anti-windup problem, which is amenable of a global, robust-in-the-large solution. The performance-robustness trade-off involved in the definition of the relaxed problem consists in imposing (a) only in nominal operating conditions, meanwhile requiring (b) in a robustin-the-large fashion. It is important to remark that neither approach is strictly better than the other, as actually each one can be preferable to the other depending on the specific application. In particular, the former approach is better suited for cases where non negligible (though sufficiently small) uncertainty is always present on the plant, so that performance must (and can) be guaranteed in a robust fashion; on the other hand, if the nominal model of the plant is an accurate description in most operating conditions, but large uncertainties (possibly impairing the achievement of robust performance) may appear from time to time, achieving nominal performance and robust stability is enough to yield a high performance control system. A more in-depth discussion on this topic is found in (Galeani and Teel, 2004).

The state-feedback solution of the relaxed problem proposed in (Galeani and Teel, 2004) is a modification of the $\mathcal{L}_{2}$ anti-windup compensator of (Teel and Kapoor, 1997a), obtained by merging the basic recipe for "uniting local and global controllers" proposed in (Teel and Kapoor, 1997b) with ideas from "two degrees of freedom" (Grimble, 1994) and "model following" (Tyler, 1964) controllers, and especially (Moore et al., 1986), which shows how a high performance controller can be "robustified" by means of another robust controller. The main drawback of the solution given in (Galeani and Teel, 2004) consists in its inherently state-feedback nature; in fact, its implementation by output feedback requires rather restrictive conditions (Galeani, 2004).

The previous discussion motivated the interest for the relaxed anti-windup problem, and for the quest for existence conditions for output feedback solutions to the problem. This paper provides a partial answer to the question above, by showing that such solutions always exist at least for the class of additively perturbed systems. Such a class of uncertain systems has been widely studied and is particularly relevant in applications for several reasons, among which the fact that identification procedures often yield uncertain model sets described by a nominal system and a (possibly frequency shaped) set of additive perturbations (see e.g. (Milanese and Taragna, 2002) and references therein). Remarkably, the availability of frequency dependent bounds on the magnitude of the additive uncertainty can be exploited in the design of the anti-windup compensators proposed in this paper. In particular, the knowledge of (possibly tight) frequency dependent bounds for the additive uncertainty can be exploited in the performance/robust-stability trade-off in order to limit the performance loss due to off-nominal operating conditions.

\section{Notation}

For a given convex set $\mathcal{U} \subset \mathbb{R}^{p}$ and a vector $u \in \mathbb{R}^{p}$, let $\operatorname{dist}_{\mathcal{U}}(u):=\inf _{w \in \mathcal{U}}(|u-w|)$, where $|\cdot|$ represents the Euclidean norm; $\operatorname{int}(\mathcal{U})$ is the interior of $\mathcal{U}$. Given two vectors $x$ and $y$, their stacking $\left[\begin{array}{ll}x^{\prime} & y^{\prime}\end{array}\right]^{\prime}$ will be denoted $(x, y)$. The $\mathcal{L}_{2}$ norm of a signal $w(\cdot)$ is defined as $\|w\|_{2}:=\sqrt{\int_{0}^{\infty}|w(t)|^{2} d t}$, and $w \in \mathcal{L}_{2}$ if $\|w\|_{2}<\infty$. A system $\Sigma$ with input $(u, v)$ and output $(y, z)$ is said to have finite incremental $\left(\mathcal{L}_{2}\right.$ induced) gain $\gamma_{y, u}^{(\Sigma)} \in \mathbb{R}_{\geq 0}$ from $u$ to $y$ if for any initial condition $\sigma_{0}$, any input $v(\cdot)$, and any pair of inputs $u_{1}(\cdot), u_{2}(\cdot)$, it holds that

$\left\|y\left(\cdot ; \sigma_{0}, u_{1}, v\right)-y\left(\cdot ; \sigma_{0}, u_{2}, v\right)\right\|_{2} \leq \gamma_{y, u}^{(\Sigma)}\left\|u_{1}-u_{2}\right\|_{2}$, where $y\left(t ; \sigma_{0}, u, v\right)$ is the output response at time $t$ to the initial condition $\sigma_{0}$ and inputs $u(\cdot), v(\cdot)$. If $\Sigma$ is linear time invariant (LTI) with transfer function $W(s)$, its incremental gain is equal to $\|W(s)\|_{\infty}:=\sup _{\omega \in \mathbb{R}} \bar{\sigma}(W(j \omega))$, where $\bar{\sigma}(\cdot)$ is the maximum singular value of the argument. The trivial system (whose output is identically null for any input) is indicated by 0 , and has zero incremental gain.

\section{PROBLEM DATA}

Uncertain plants $P_{\Psi}$, connection of a nominal model $P$ and a "perturbation" $\Psi$, are considered:

$$
\begin{aligned}
& \dot{x}=A x+B_{2} u, \\
& z=C_{1} x+D_{11} d+D_{12} u+z_{\Psi}, \\
& y=C_{2} x+D_{21} d+D_{22} u+y_{\Psi},
\end{aligned}
$$

with measured output $y \in \mathbb{R}^{q}$, performance output $z$, control input $u \in \mathbb{R}^{p}$, exogenous disturbance $d$, while $y_{\Psi}$ and $z_{\Psi}$ are the outputs of a perturbation $\Psi \in \mathcal{S}$, where $\mathcal{S}$ is a set of incrementally stable systems with $\Psi \in \mathcal{S}$ given by

$$
\begin{aligned}
\dot{\psi} & =f_{\Psi}(\psi, u), \\
y_{\Psi} & =h_{1, \Psi}(\psi, u), \\
z_{\Psi} & =h_{2, \Psi}(\psi, u),
\end{aligned}
$$

(different elements of $\mathcal{S}$ can have different state spaces). $0 \in \mathcal{S}$ is assumed, so that $P_{0}=P$. Notice that there is no loss of generality, from the input/output and stability point of view, in considering the disturbance $d$ as not affecting 
(1a), since (1) is linear and (for the sake of a global discussion) will be later assumed to be asymptotically stable: under such conditions, the effects of a disturbance $\bar{d}$ affecting all three equations (1) can be represented as the effect of $d$, only affecting (1b) and (1c), with $d$ a filtered version of $\bar{d}$.

Let $\mathcal{S}_{\rho}:=\left\{\Psi \in \mathcal{S}: \gamma_{y_{\Psi}, u_{\Psi}}^{(\Psi)}<\rho\right\}$ for $\rho \in \mathbb{R}_{>0}$, i.e. $\mathcal{S}_{\rho} \subset \mathcal{S}$ contains only uncertainties with incremental gain less than $\rho$ from $u_{\Psi}=u$ to $y_{\Psi}$. A property (e.g., $\mathcal{L}_{2}$ stability) possibly enjoyed by a system $\Sigma_{\Psi}$ with a parameter $\Psi \in \mathcal{S}$ is nominal if enjoyed by $\Sigma_{\Psi}$ when $\Psi=0$, it is robust in the small (with respect to $\mathcal{S}$ ) if enjoyed by $\Sigma_{\Psi}$ for all $\Psi \in \mathcal{S}_{\rho}$ for some $\rho \in \mathbb{R}_{>0}$, it is robust-in-the-large (with respect to $\mathcal{S}$ ) if enjoyed by $\Sigma_{\Psi}$ for all $\Psi \in \mathcal{S}$.

In the anti-windup (aw) problem, a controller $K_{M}$ (assumed to be linear for simplicity) designed for system (1) is supposed to be given:

$$
\begin{aligned}
\dot{x}_{c} & =A_{c} x_{c}+B_{c} u_{c}+E_{c} r, \\
y_{c} & =C_{c} x_{c}+D_{c} u_{c}+F_{c} r,
\end{aligned}
$$

(here, $r$ is a reference signal) and the goal of aw synthesis is to design an additional aw compensator $K_{A W}$ of the form

$$
\begin{aligned}
\dot{x}_{a w} & =f_{a w}\left(x_{a w}, y, y_{c}\right), \\
v_{1} & =h_{a w, 1}\left(x_{a w}, y, y_{c}\right), \\
v_{2} & =h_{a w, 2}\left(x_{a w}, y, y_{c}\right),
\end{aligned}
$$

which, suitably connected to $P_{\Psi}$ and $K_{M}$, ensures some nice properties for the overall closed loop system. Since it will be useful to have shorthand notations to refer to different interconnections of $P_{\Psi}, K_{M}$ and $K_{A W}$, define the following closed loop systems (cls): (1), (2), (3) form the unconstrained cls $\Sigma_{U}$ when $u=y_{c}, u_{c}=y$, and the saturated cls $\Sigma_{S}$ when $u=\operatorname{sat}\left(y_{c}\right), u_{c}=y$; (1), (2), (3), (4) form the unconstrained aw cls $\Sigma_{U A W}$ when $u=y_{c}+v_{1}, u_{c}=y+v_{2}$, and the (saturated) aw cls $\Sigma_{S A W}$ when $u=\operatorname{sat}\left(y_{c}+v_{1}\right)$, $u_{c}=y+v_{2}$. Different "hats" denote a signal related to a system (e.g., the state $x$ of $P$ ) in a cls: - denotes the signal in $\Sigma_{U}$ (e.g., $\bar{x}$ for the state of $P$ as a subsystem of $\left.\Sigma_{U}\right)$, $\sim$ denotes the signal in $\Sigma_{U A W}$ (e.g., $\tilde{x}$ for the state of $P$ as a subsystem of $\left.\Sigma_{U A W}\right)$, and no hat denotes the signal in $\Sigma_{S A W}$ (e.g., $x$ for the state of $P$ as a subsystem of $\left.\Sigma_{S A W}\right)$.

Following (Teel and Kapoor, 1997a), $\sigma: \mathbb{R}^{p} \rightarrow$ $\mathbb{R}^{p}$ is a "saturation" function if $\exists \mathcal{U} \subset \mathbb{R}^{p}, \mathcal{U}$ compact and convex, and $\exists L, b \in \mathbb{R}_{>0}$ such that $[\sigma(u+\varphi)-(u+\varphi)] \leq L u^{\prime}(\sigma(u+\varphi)-\varphi)$ and $|\sigma(u+w)-\sigma(u)| \leq \min \{L|w|, b\}, \forall u, w \in \mathbb{R}^{p}$, $\forall \varphi \in \mathcal{U}$. The "standard" decoupled saturation function $y_{i}=\operatorname{sign}\left(u_{i}\right) \min \left\{\left|u_{i}\right|, u_{i, M A X}\right\}, i=$ $1, \ldots, p$, satisfies the above properties if $\mathcal{U}$ is chosen as any compact and convex subset of the interior of its linear region.

\section{A WEAKENED GLOBAL $\mathcal{L}_{2} \mathrm{AW}$ PROBLEM}

The main difference between a usual anti-windup problem and the weakened problem of interest in this paper consists in the fact that in the weakened problem the overall closed loop response from $d$ to $z$ is allowed to be modified in order to be able to robustify the aw closed loop system with respect to a larger class of uncertainties. Intuitively, such a trade off is needed since both $d$ and $y_{\Psi}$ are not measured (so that their contributions in (1c) are not distinguishable), and then any "filtering" action performed on $y_{\Psi}$ in order to enhance robustness also affects the overall response to $d$, thus modifying the response from $d$ to $z$ even for $\Psi=0$. While the interested reader is referred to (Galeani and Teel, 2004) for a thorough discussion on the relation between the global $\mathcal{L}_{2}$ aw problem and its weakened version, the definition and solution of the original $\mathcal{L}_{2}$ aw problem in (Teel and Kapoor, 1997a) is recalled next for completeness (in a form restricted to the issues of interest here), and in order to enable the reader to understand the underlying basic issues. As a last remark, the reader is warned about the fact that the cited issues are not due to the solution of the aw problem proposed in (Teel and Kapoor, 1997a), but to the very "natural" definition of $\left(\mathcal{L}_{2}\right)$ aw, so that if the specific aw problem arising in a given application is prone to the considered robustness issues, any solution (independently from the approach used in its synthesis) to such a problem will suffer from the same issues.

For the global $\mathcal{L}_{2}$ aw problem to make sense, the following assumption is needed.

Assumption 1. System $\Sigma_{U}$ is well-posed and internally stable for all $\Psi \in \mathcal{S}$.

Definition 2. The robust (in the small), $\mathcal{L}_{2}$ aw problem for $\mathcal{U} \subset \mathbb{R}^{p}$ and $\mathcal{S}$ is to find an aw compensator such that $\Sigma_{S A W}$ is well-posed and

(1) if $x_{a w}(0)=0$ and $\bar{u}(\cdot) \equiv \operatorname{sat}(\bar{u}(\cdot))$ then $z(\cdot) \equiv \bar{z}(\cdot)$

(2) if $\operatorname{dist}_{\mathcal{U}}(\bar{u}(\cdot)) \in \mathcal{L}_{2}$ then $(z-\bar{z})(\cdot) \in \mathcal{L}_{2} ;$

for all $\Psi \in \mathcal{S}_{\rho}$ with sufficiently small $\rho$.

A sufficient condition, adapted from (Teel and Kapoor, 1997a), for the solution of the global $\mathcal{L}_{2}$ aw problem is as follows.

Theorem 3. Under Assumption 1, the problem in Definition 2 is solvable if $A$ is Hurwitz, and a possible aw compensation is:

$$
\begin{aligned}
\dot{x}_{a w} & =A x_{a w}+B_{2}\left[\operatorname{sat}\left(y_{c}+v_{1}\right)-y_{c}\right] ; \\
v_{1} & =-B_{2}^{\prime} P x_{a w} ; \\
v_{2} & =-C_{2} x_{a w}-D_{22}\left[\operatorname{sat}\left(y_{c}+v_{1}\right)-y_{c}\right] ;
\end{aligned}
$$

with $P \in \mathbb{R}^{n \times n}, P=P^{\prime}>0: A^{\prime} P+P A<0$. 
For the global weakened $\mathcal{L}_{2}$ aw problem to make sense, different assumptions are needed.

Assumption 4. System $\Sigma_{U}$ is well-posed and internally stable for $\Psi=0$.

Compared to Assumption 1, Assumption 4 requires $\Sigma_{U}$ to be only nominally stable, so that $K_{M}$ can be designed focusing on nominal performance, disregarding robust stability issues.

Assumption 5. $A$ is Hurwitz, all $\Psi \in \mathcal{S}$ have a finite incremental gain from $u$ to $\left(y_{\Psi}, z_{\Psi}\right)$, and there exists a $\gamma \in \mathbb{R}_{>0}$ such that $\mathcal{S}_{\gamma}=\mathcal{S}$.

Since no global result can be obtained for exponentially unstable controlled plants with bounded controls, Assumption 5 is quite mild in the present context. Moreover, Assumption 5 is coherent with the kind of nominal models and perturbation sets obtained in several approaches to identification,

Definition 6. The weakened global $\mathcal{L}_{2}$ aw problem for $\mathcal{U}$ with domain of robustness $\mathcal{S}$ is to find an aw compensator such that $\Sigma_{S A W}$ is well-posed and

(1) for $\Psi=0$ and $d=0, \exists x_{a w}^{0}$ : if $x_{a w}(0)=x_{a w}^{0}$ and $\bar{u}(\cdot) \equiv \operatorname{sat}(\bar{u}(\cdot))$, then $z(\cdot) \equiv \bar{z}(\cdot)$

(2) $\Sigma_{U A W}$ is well-posed and internally stable;

(3) if $\operatorname{dist}_{\mathcal{U}}(\tilde{u}(\cdot)) \in \mathcal{L}_{2}$ then $(z-\tilde{z})(\cdot) \in \mathcal{L}_{2}$.

for all $\Psi \in \mathcal{S}$.

A comparison is in order between Definition 6 and Definition 2. Item 1 in Definition 6 requires that, for references and initial conditions such that saturation never activates in $\Sigma_{S}$, the nominal $(\Psi=0, d=0)$ behaviour from $r$ to $z$ of $\Sigma_{S A W}$ matches the corresponding behaviour of $\Sigma_{U}$; on the other hand, item 1 in Definition 2 requires the stronger property that for all $\Psi$ having sufficiently small incremental gain, for all signals $(r, d)$ and initial conditions such that the saturation never activates in $\Sigma_{S}$, the response from $(r, d)$ to $z$ in $\Sigma_{S A W}$ matches the corresponding response in $\Sigma_{U}$.

As for item 3 of Definition 6, similar to item 2 of Definition 2, the effectiveness of the aw is assessed by bounding the $\mathcal{L}_{2}$ difference between the responses of $\Sigma_{S A W}$ and another system which is well-behaved robustly with respect to $\mathcal{S}$; however, contrary to what is done in item 2 of Definition 2 (also, compare Assumption 1 to Assumption 4), the comparison system here is $\Sigma_{U A W}$ and not $\Sigma_{U}$ (which needs not even to be stable for all $\Psi \in$ $\mathcal{S}$; instead, robust well-posedness and stability of $\Sigma_{U A W}$ is required in item 2 of Definition 6 ). A positive consequence of not requiring robust stability of $\Sigma_{U}$ is to decouple the problem of guaranteeing robustness from the design of controller $K_{M}$ in (3), which can then be focused on performance. The additional item 2 of Definition 6 is then clearly motivated by the need to ensure that the saturating motions of $\Sigma_{S A W}$ are compared with the motion of a system which is stable and wellposed robustly in the large with respect to $\mathcal{S}$.

\section{MAIN RESULT}

In the following theorem, $F=\left(A_{F}, B_{F}, C_{F}, D_{F}\right)$ is a $q$-input, $q$-output asymptotically stable LTI system with transfer matrix $F(s)$.

Theorem 7. If Assumption 4 and Assumption 5 hold, $\exists \gamma_{K}^{*} \in \mathbb{R}_{>0}, \exists \gamma_{F}^{*}(\cdot): \mathbb{R}_{\geq 0} \rightarrow \mathbb{R}_{\geq 0}$ such that $\forall K \in \mathbb{R}^{p \times n}$ satisfying $\bar{\sigma}(\bar{K})<\gamma_{K}^{*}, \forall \omega \in$ $\mathbb{R}_{\geq 0}$, and $\forall F(s)$ satisfying $\bar{\sigma}(F(j \omega))<\gamma_{F}^{*}(\omega)$, $\forall \omega \in \mathbb{R}_{\geq 0}$, the LTI aw compensation $K_{A W}=$ $\left(A_{a w}, B_{a w}, C_{a w}, D_{a w}\right)$ with state $x_{a w} \in \mathbb{R}^{2 n+n_{F}}$, input $u_{a w}=\left(\operatorname{sat}\left(y_{c}+v_{1}\right), y, y_{c}\right)$, output $y_{a w}=$ $\left(v_{1}, v_{2}\right)$,

$$
\begin{aligned}
& {\left[\begin{array}{c|c}
A_{a w} & B_{a w} \\
\hline C_{a w} & D_{a w}
\end{array}\right]=} \\
& =\left[\begin{array}{ccc|ccc}
A & 0 & 0 & B_{2} & 0 & 0 \\
-B_{F} C_{2} & A_{F} & 0 & -B_{F} D_{22} & B_{F} & 0 \\
0 & 0 & A & 0 & 0 & B_{2} \\
\hline-K & 0 & K & 0 & 0 & 0 \\
-D_{F} C_{2} & C_{F} & C_{2} & -D_{F} D_{22} & D_{F}-I & D_{22}
\end{array}\right]
\end{aligned}
$$

solves the problem in Definition 6.

Remark 8. The aw compensator in Theorem 7 has two main differences with respect to the one in (Galeani and Teel, 2004): first, it does not reduce to the $\mathcal{L}_{2}$ aw compensator in (Teel and Kapoor, 1997a), i.e. (5), for a suitable choice of $F$; second, it requires no state measurements. Clearly, the second feature is extremely important from the point of view of implementation.

As for the relation of the proposed aw compensator with (5), if no disturbances are present, it is possible to define a "corresponding" $\mathcal{L}_{2}$ aw compensator in the following sense: the response induced in nominal conditions by the proposed aw compensator (if suitably initialized) is the same response induced by (5) with (5b) replaced by $v_{1}=-K x_{a w}$.

The proof of Theorem 7 is omitted due to space constraints; it is based on a small gain reasoning and is similar to the proof of Theorem 3 , by partitioning the state of $K_{A W}$ as $x_{a w}^{\prime}=\left[\begin{array}{lll}x_{S}^{\prime} & x_{F}^{\prime} & x_{M}^{\prime}\end{array}\right]$ (with $x_{S} \in \mathbb{R}^{n}, x_{F} \in \mathbb{R}^{n_{F}}, x_{M} \in \mathbb{R}^{n}$ ) and rewriting $\Sigma_{S A W}$ as:

$$
\begin{aligned}
& P_{S}:\left\{\begin{array}{l}
\dot{x}_{S}=A x_{S}+B_{2} u, \\
y_{S}:=C_{2} x_{S}+D_{22} u,
\end{array}\right. \\
& F:\left\{\begin{array}{l}
\dot{x}_{F}=A_{F} x_{F}+B_{F}\left(y-y_{S}\right), \\
y_{F}:=C_{F} x_{F}+D_{F}\left(y-y_{S}\right),
\end{array}\right. \\
& P_{M}:\left\{\begin{array}{l}
\dot{x}_{M}=A x_{M}+B_{2} y_{c}, \\
y_{M}:=C_{2} x_{M}+D_{22} y_{c},
\end{array}\right.
\end{aligned}
$$




$$
\begin{gathered}
K_{M}:\left\{\begin{array}{l}
\dot{x}_{c}=A_{c} x_{c}+B_{c}\left(y_{M}+y_{F}\right)+E_{c} r \\
y_{c}=C_{c} x_{c}+D_{c}\left(y_{M}+y_{F}\right)+F_{c} r
\end{array}\right. \\
P:\left\{\begin{array}{l}
\dot{x}=A x+B_{2} u, \\
z=C_{1} x+D_{11} d+D_{12} u+z_{\Psi}, \\
y=C_{2} x+D_{21} d+D_{22} u+y_{\Psi}, \\
u=\operatorname{sat}\left(y_{c}+y_{K}\right) .
\end{array}\right.
\end{gathered}
$$

Remark 9. With respect to the compensator in (Galeani and Teel, 2004), the need for state measurements is avoided by using the estimates $x_{S}$ of $x$, at the price of introducing $n$ additional states in $K_{A W}$.

Remark 10. Comparing the interconnection condition $u_{c}=y+v_{2}$ and (10), it is clear that $v_{2}=-y+y_{M}+y_{F}$, so that the unconstrained controller $K_{M}$ is not directly fed by the output of the process. For implementation, it is more convenient to realize $u_{c}=y+v_{2}$ directly as $u_{c}=y_{M}+y_{F}$.

Remark 11. As suggested in (Galeani and Teel, 2004), standard loop-shaping techniques can be used to determine suitable $K$ 's and $F$ 's (whose existence is guaranteed by Theorem 7); a detailed discussion on this issue is omitted due to space constraints. However, it is worth at least remarking that minimizing $\|I-F(s)\|_{\infty}$ makes the response of $\Sigma_{U A W}$ closer to the response of $\Sigma_{U}$ (thus reducing the amount of aw performance traded for robust stability in the weakened aw problem), whereas a suitable choice of $K$ yields faster recovery after saturation occurs.

\section{AN EXAMPLE}

Consider the nominal mass-spring-damper system

$$
\begin{aligned}
& \dot{x}=A x+B_{2} u=\left[\begin{array}{cc}
0 & 1 \\
-k / m & -f / m
\end{array}\right] x+\left[\begin{array}{c}
0 \\
1 / m
\end{array}\right] u, \\
& y=C_{2} x=\left[\begin{array}{ll}
1 & 0
\end{array}\right] x,
\end{aligned}
$$

having state $x^{\prime}=[q \dot{q}]$ (where $q$ and $\dot{q}$ are the position and the speed of the body attached to the spring) for which $m=0.1, k=1, f=$ 0.001 . Since the poles of the system are very underdamped, a two degrees of freedom controller (such that $y_{c}(s)=C_{f b}(s)\left(C_{f f}(s) r-u_{c}(s)\right)$, with $C_{f b}(s)=200 \frac{(s+5)^{2}}{s(s+80)}$ and $\left.C_{f f}(s)=\frac{5}{2 s+5}\right)$ is given which induces a quickly convergent response, asymptotic tracking of step references and rejection of step disturbances. In the presence of input saturation, the saturated closed loop response dramatically deteriorates, showing the need for anti-windup compensation. The open loop, unconstrained closed loop, saturated closed loop responses in nominal conditions are shown in Fig. 1; the performance recovery achievable in nominal conditions by using the weakened aw compensator (equivalently, by using its "corresponding" $\mathcal{L}_{2}$ aw compensator; see Remark 8) is clear from Fig. 1.

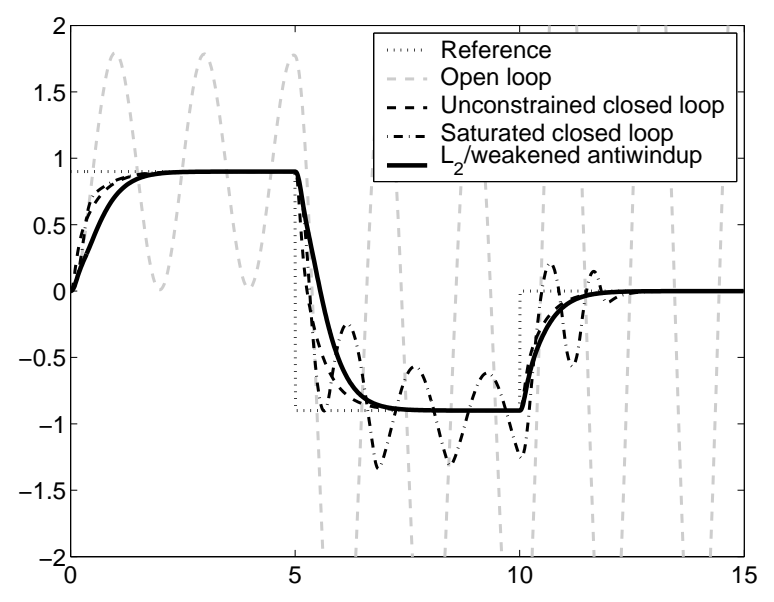

Fig. 1. Nominal $(\psi=0)$ output responses. The $\mathcal{L}_{2}$ aw and the weakened aw outputs coincide.

Consider a real actuator with transfer function $V(s)=\frac{a}{s+a}$, with the parameter $a$ usually ranging in the set $[100, \infty)$ but occasionally dropping in the set $a \in[10,100)$ due to particularly unfavourable operating conditions. The use of the real actuator (instead of the ideal $V_{0}(s)=1$ ) introduces a set of input multiplicative perturbations $\mathcal{M}:=\left\{\mu(s): \mu(s)=\frac{-s}{s+a}, a \in[10, \infty)\right\} \cup\{0\}$. The set $\mathcal{M}$ can be recast as a set of additive perturbations $\mathcal{S}:=\left\{\Psi(s): \Psi(s)=P_{0}(s) \frac{-s}{s+a}, a \in\right.$ $[10, \infty)\} \cup\{0\}$, where $P_{0}(s):=C_{2}(s I-A)^{-1} B_{2}$. The upper bound $|\Psi(j \omega)|<\bar{\psi}(\omega):=\left|\frac{j \omega}{j \omega+10}\right|=$ $\frac{\omega}{\sqrt{\omega^{2}+100}}$ holds $\forall \Psi \in \mathcal{S}$. For $a<a_{\text {min }} \approx 17.4$, the unconstrained closed loop system $\Sigma_{U}$ becomes unstable, so that for such values of $a$ any nonweakened form of anti-windup is not applicable.

The parameter $K=10^{-3}[0.055-605]$ is obtained as $K=-\rho B^{\prime} Q$, with $Q$ solution of $A Q+$ $Q A^{\prime}=-\left[\begin{array}{ll}.1 & .51 \\ .51 & 10\end{array}\right]$, and $\rho$ chosen in such a way to minimize the maximum real part of the eigenvalues of $A+K B$, while guaranteeing a $\mathcal{L}_{2}$ induced gain smaller than 1.1 for the negative feedback closed loop having the saturation in the forward

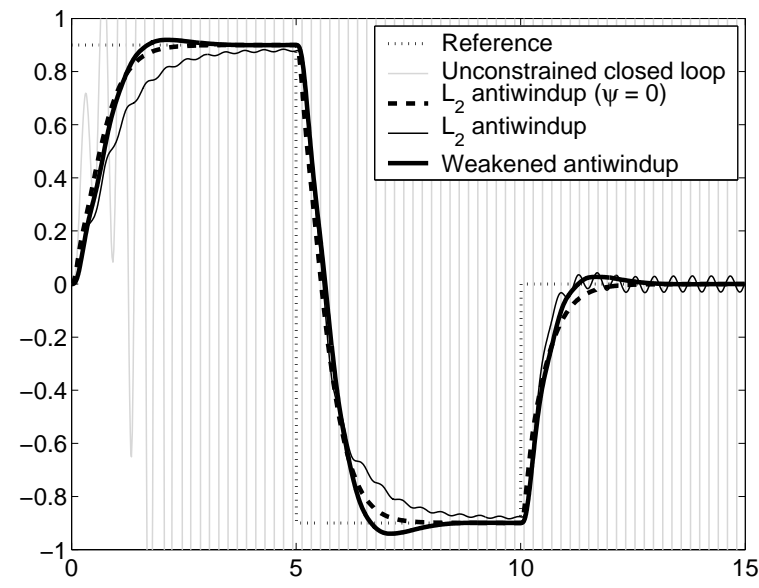

Fig. 2. Perturbed $(a=10)$ output responses. The weakened aw output is close to the nominal $\mathcal{L}_{2}$ aw, while the other responses are unstable. 


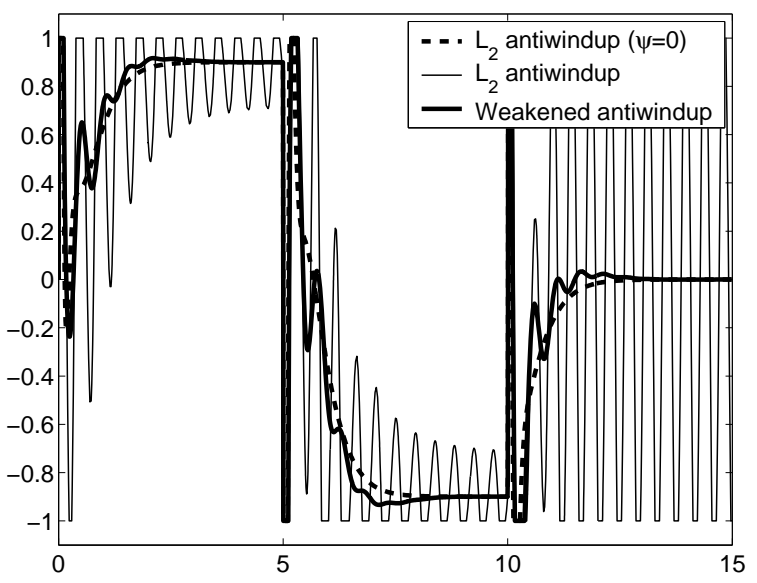

Fig. 3. Plant input for $(a=10)$. Limits cycles show up if non-weakened aw is applied.

path and $K(s I-A)^{-1} B$ in the feedback path. The choice $F(s)=\frac{10 s^{2}+.1 s+100}{s^{3}+16 s^{2}+70 s+100}$ yields

$$
\bar{\psi}(\omega) \bar{\sigma}((I+K G(j \omega)) T(j \omega) F(j \omega))<(1.1)^{-1},
$$

$\forall \omega \in \mathbb{R}_{\geq 0}$, where $T(s)$ is the transfer matrix from input $y_{F}$ to output $y_{c}$ of the closed loop formed by (8) and (10), and $G(s):=(s I-A)^{-1} B_{2}$. For comparison, in simulations the "corresponding" $\mathcal{L}_{2}$ aw compensator (5) with $v_{1}=-K x_{a w}$ (not satisfying the small gain condition above) is considered.

Fig. 2 shows the response of the weakened aw in presence of the severest perturbation in $\mathcal{S}$ $(a=10)$, which is quite close to the nominal aw response in Fig. 1, thus showing that the "aw performance" loss due to the weakened formulation of the aw problem is rather mild in this example. Fig. 2 also shows that the unconstrained closed loop is unstable for $a=10$, so that any nonweakened form of aw is not applicable for such large parameter variations; in fact the response with the "corresponding" $\mathcal{L}_{2}$ aw compensator (i.e. (5) with $v_{1}=-K x_{a w}$ ) for $a=10$ is characterized by persistent oscillations, especially evident in the last 5 seconds of simulation in the output in Fig. 2, and in the input in Fig. 3.

\section{CONCLUSIONS}

An output feedback solution to the weakened antiwindup problem for additively perturbed plants has been proposed, thus overcoming the need for state feedback for this class of uncertain systems.

Future work will focus on optimal design, adaptive and order reduction for the proposed anti-windup compensation. Moreover, it would be interesting to characterize under which conditions the "antiwindup performance" loss due to the relaxed formulation of the anti-windup problem is negligible (as in the example in this paper).

\section{REFERENCES}

Galeani, S. (2004). On output feedback robustified anti-windup compensators. In: Proc. $12^{\text {th }}$ Med. Conf. on Control and Automation.

Galeani, S. and A.R. Teel (2004). On performance and robustness issues in the antiwindup problem. In: Proc. $43^{\text {rd }}$ IEEE Conf. on Decision and Control.

Grimble, M. J. (1994). Two and a half degrees of freedom LQG controller and application to wind turbines. IEEE Trans. Aut. Contr. 39, 122-127.

Grimm, G., A.R. Teel and L. Zaccarian (2004). Robust linear anti-windup synthesis for recovery of unconstrained performance. Int. J. Robust Nonlinear Control 14, 1133-1168.

Grimm, G., J. Hatfield, I. Postlethwaite, A.R. Teel, M.C. Turner and L. Zaccarian (2003). Antiwindup for stable linear systems with input saturation: an LMI-based synthesis. IEEE Trans. Aut. Contr. 48, 1509-1525.

Hanus, R. (1988). Antiwindup and bumpless transfer: a survey. In: Proceedings of the 12th IMACS World Congress. Vol. 2. Paris, France. pp. 59-65.

Kothare, M. V., P. J. Campo, M. Morari and C. N. Nett (1994). A unified framework for the study of antiwindup designs. Automatica 30, 1869-1883.

Milanese, M. and M. Taragna (2002). Optimality, approximation, and complexity in set membership $H_{\infty}$ identification. IEEE Trans. Aut. Contr. 47, 1682-1690.

Moore, J.B., L. Xia and K. Glover (1986). On improving control-loop robustness of modelmatching controllers. System \& Control Letters 7, 83-87.

Mulder, E. F., M. V. Kothare and M. Morari (2001). Multivariable antiwindup controller synthesis using linear matrix inequalities. $A u$ tomatica 37, 1407-1416.

Peng, Y., D. Vrančić, R. Hanus and S. S. R. Weller (1998). Anti-windup designs for multivariable controllers. Automatica 34, 1559-1565.

Saeki, M. and N. Wada (2002). Synthesis of a static anti-windup compensator via linear matrix inequalities. Int. J. Robust Nonlinear Control 12, 927-953.

Teel, A.R. and N. Kapoor (1997a). The $\mathcal{L}_{2}$ antiwindup problem: Its definition and solution. In: Proc. 4th European Control Conf.

Teel, A.R. and N. Kapoor (1997b). Uniting local and global controllers. In: Proc. 4th European Control Conf.

Turner, M.C., G. Herrmann and I. Postlethwaite (2004). Accounting for uncertainty in antiwindup synthesis. In: American Control Conference.

Tyler, J. Jr. (1964). The characteristics of modelfollowing systems as synthesized by optimal control. IEEE Trans. Aut. Contr. 9, 485-498. 